\title{
PRODUCTOS NATURALES: UTILIDAD Y MEDIDA DE SU EFICACIA EN COSMÉTICA
}

\author{
Astrid de Castro* \\ *Facultad de Farmacia. Universidad Central de Venezuela
}

\section{RESUMEN}

Teniendo presente la preferencia del consumidor por materiales naturales o biotecnológicos en cosmética, nos propusimos estudiar algunas materias primas naturales que pudieran constituir una alternativa válida, segura y eficaz. Para ello se estudió: El efecto hidratante de la proteína de soja. Productora de fitosteroles y con efecto inhibidor de las proteinasas (1), La actividad antipruriginosa del residuo lipídico de la cebada. (2), La efectividad de un Protector solar con Oxido de Zinc, Dióxido de titanio (bloqueadores), Complejo de Proteína de soja (hidratante, antiirritante, con actividad antiozono) y residuo lipídico de la cebada como antipruriginoso. Se diseñaron varias formulaciones siguiendo los principios básicos (3), y en el caso de la proteína de soja, se comparó el efecto con Aloe vera, el residuo lipídico de la cebada se comparó con un placebo con aloe vera y manzanilla, y finalmente la efectividad del Protector solar se determinó mediante un estudio clínico. En todos los casos se realizaron estudios in vivo no invasivos. Los resultados obtenidos demuestran que: la proteína de soja mantiene el efecto hidratante mayor tiempo que el Aloe vera,el residuo lipídico de la cebada tiene actividad antipruriginosa significativamente superior al de la mezcla de cebada y manzanilla .La formulación del Protector solar ofrece una alternativa segura y efectiva en el tratamiento de la piel dañada, sensible y delicada.

Palabras clave: Productos naturales, cosmética.

\begin{abstract}
Having in mind the preference of consumers for natural or biotecnological materials in cosmetics, we decided to study some natural raw materials that could constitute a valid, safe and efficient alternative. To do so, we studied: first the moisturizing effect of soja protein fitosterol producer and with inhibitory effect on proteinases (1); second the antipruriginous activity of lipidic residue of barley (2), and third, the effectivity of a sun protector with zinc dioxide, titanium dioxide (blockers), soja protein complex (moisturizing anti-iritative with anti-ozone activity) and lipidic residue of barley as antipruriginous. Several formulationes were designed according to some basic principles(3); and in case of soja protein, the effect was compared with aloe vera, the lipidic residue of cebada has been compared with a placebo, aloe vera and chamomile and finally the effectivity of the sun protector was determined by means of a clinical study.

In all cases, non invasive in vivo studies were performed. The results obtained demostrate that: the soja protein hold up the moisturizing effect for more time than aloe vera.

The lipidic residue of Barley has a better antipruriginous effect, than the mix of barley and chamomile. The formulation of sun protector offers a safe and effective alternative for the treatment of damaged, sensible and delicate skin.
\end{abstract}

Key words: Natural materials, cosmetics 


\section{INTRODUCCION}

La preferencia de los consumidores por el uso de materiales, así como los obtenidos por procesos biotecnológicos, a los de origen animal o químico en productos para el cuidado de la piel, obedece al hecho de que por una parte se busca evitar las posibles reacciones adversas, y por la otra, ellas constituyen fuentes renovables de materias prima. Pero a la vez, se recomienda la purificación de los mismos para asegurar la estabilidad, seguridad, la baja toxicidad y eficacia de sus efectos.

Infinidad de productos naturales han sido extensamente utilizados en la fabricación de productos cosméticos, ya que se dice que son de obtención rápida, fácil y de bajo costo. El contenido de agua en la piel es más importante para su flexibilidad, que el contenido de grasas. Cuando el estrato córneo se encuentra deshidratado, pierde su flexibilidad y la tensión mecánica puede producir sensación de rigidez y conducir a la aparición de grietas, mientras que la apariencia hidratada $y$ saludable de la piel puede influenciarse por la aplicación externa de productos conocidos como humectantes y emolientes.

La proteína de soja representa una materia prima de origen natural adecuada para la producción comercial de fitosteroles, y que por su similitud con el colesterol le permite comportarse en forma parecida, siendo un derivado de origen vegetal. Se ha reportado que el uso de fitosteroles mejora la tonicidad de la piel de los individuos expuestos a la radiación solar, y reduce el prurito, la descamación, la quemadura, el enrojecimiento, el calor y las ampollas. Este efecto puede compararse con el de ungüentos con $1 \%$ de hidrocortisona.(4).

Los fitosteroles no pueden ser sintetizados por organismos animales. Teniendo esto presente, resulta sorprendente encontrar bajas concentraciones de estas sustancias en la piel humana. Los fitosteroles a diferencia del colesterol, deben estar incorporados dentro de la membrana basal y alcanzan la superficie celular cutánea durante la diferenciación (5).

La semilla de soja tiene la siguiente composición: $16-22 \%$ de aceite, $39 \%$ de albúmina, $5.7 \%$ de fibras crudas, $25 \%$ de hidratos de carbono, 5-6\% de ceniza. Adenina, guanina, histidina, arginina, trigonelina, colina. Glucosidos: $A=$ genistidina: $\mathrm{B}=$ daidcina. $\quad \mathrm{C}, \mathrm{C} 1, \quad \mathrm{C} 2=$ glucósidos $\mathrm{de}$ saponina, un $12 \%(6)$.

También se ha reportado que el extracto acuoso de la proteína de soja, es un inhibidor de las proteinasas (elastasa y triptasa) que juegan un papel importante en los procesos de envejecimiento de la piel, y por lo tanto es de utilidad para mantener la elasticidad, tersura, suavidad y para aumentar la hidratación de la piel. La inhibición de la elastasa de los leucocitos y de la elastasa de los fibroblastos es de gran significación en la prevención de la irritación e inflamación de la piel, causadas por una excesiva exposición Mal sol, productos químicos y factores ambientales (7).

Por otra parte, debemos reconocer que ciertas situaciones como la exposición exagerada a la radiación solar, el rasurado, así como la aplicación de ciertos productos cosméticos sobre pieles sensibles y delicadas, está acompañada muy frecuentemente de irritación y prurito, por lo tanto es de suma importancia desarrollar formulaciones cosméticas que sean de utilidad para contrarrestar esta sensación.

E1 prurito, picazón o comezón corresponde a una sensación cutánea especial, irritante, que induce al rascado como respuesta (8). Se le ha considerado como una unidad sensorial primaria, en lugar de una unidad subumbral del dolor, ya que clínicamente las sensaciones de dolor o prurito son fácilmente distinguibles. 
Los estímulos que pueden producirlo son muy variados, y van desde un alambre frío, la electricidad, el calor, la histamina, la quinina, las sales biliares y hasta productos cosméticos (9). La histamina es eL mediador clásico del prurito, aún cuando también puede ser causado por la sustancia $P$, las prostaglandinas, la serotonina, los leucotrienos, las proteasas, los péptidos (8).

Entre las causas del prurito relacionadas con productos cosméticos, se señalan: la exposición exagerada a la radiación solar, después del afeitado, el uso de antitranspirantes, depilatorios, despigmentantes, y con más frecuencia por el uso de jabones y fragancias.

En el tratamiento tópico del prurito se reporta el uso de: hidratación con baños refrescantes, compresas con humectantes y la aplicación local de emolientes con fenol, mentol o alcanfor (10). También se utilizan materias primas de origen natural tales como: el talco, la manzanilla, el tilo, la avena, la cebada, entre otros.

La cebada, Hordeum vulgare L. de la familia de las Gramíneas es una planta erguida que crece hasta los $120 \mathrm{~cm}$. de altura. Oriunda del Cáucaso y del Asia Occidental. Esta especie corresponde a espigas de cuatro series, cultivada en Europa y norte de Africa (6) (11). En Venezuela, corresponde a un cultivo sin importancia, ocasionalmente sembrado en los Andes. El grano, es utilizado como alimento humano y también en la elaboración de la malta y la cerveza (12).

Se ha reportado (13), que el residuo obtenido de los granos de la cebada durante el proceso de elaboración de la cerveza, posee un efecto antipruriginoso. Entre los componentes de este residuo de naturaleza lipídica, se encuentran: ácidos grasos esenciales (linoléico y linolénico), otros ácidos grasos (palmítico, mirístico, esteárico y oléico), vitaminas ( $\mathrm{B} 1$, B2, B6, B12, alfa tocoferol-Vit. E, Biotina-Vit. $\mathrm{H})$, fitosteroles (beta-sistosterol), que mejoran la estructura natural y la función de la barrera lipídica epidermal (14) (15).

Por otra parte, estudios realizados a nivel mundial, han reportado de una manera concluyente que la exposición de la piel al sol y a otras fuentes de radiación ultravioleta constituyen una de las principales causas del daño a la piel. Recientes estudios indicaron que la radicación UV es responsable de la mayoría de casos de cáncer de piel tipo nomelanoma, así como de quemaduras solares, debilitamiento del sistema inmunológico, envejecimiento prematura de la piel, etc.

El cáncer de piel ocurre hoy en día con más frecuencia que otros tipos de cáncer combinados. Se estima que en 1998 se reporten aproximadamente $10000 \quad 00$ de nuevos casos de cáncer de piel solamente en USA.

Las radiaciones correspondientes al UVB penetran hasta la epidermis, mientras que las del tipo UVA penetran más profundamente llegando hasta la dermis, produciendo daños no visibles inmediatamente, pero de acuerdo a la idiosincrasia del individuo, puede llegar a causar daños irreparables en las células del DNA H (16).

Las pantallas solares (Bloqueadores con filtros UVA, UVB y físicos) han venido siendo utilizadas para prevenir las molestas quemaduras del sol. El consumidor está en la creencia de que usando protectores solares evita la aparición del cáncer de piel, pero en la actualidad está aumentando la aparición del mismo, ya que permanecen mayor tiempo bajo la exposición solar. Por otra parte, los dermatólogos temen que aumente la aparición 
de nuevos cánceres de piel de miles de millones de personas, si los padres dejan de aplicar protectores solares a sus hijos.

Desdichadamente, las pantallas solares también inducen a la fotosenbilización química, por lo tanto debe tenerse presente que los radicales hicroxílos y otras especies de oxÍgeno reactivo, tienen el potencial de reaccionar con todos los componentes de la piel. Se han descrito (17). a los productos de la peroxidación lipídica como tóxicos, mutagénicos y carcinogénicos, así como el efecto de la reacción secundaria de las pantallas solares (18).

Cuando se indica el uso de pantallas o bloqueadores solares, se debe conocer el tipo de filtro solar y las mezclas, así como también el efecto de estos sobre la piel, de tal forma que se conozca, si son formadores de radicales de oxígeno libres (ROS) ó no, así como también es necesario conocer si en dicha formulación existen materiales capaces de no sólo de neutralizar la formación de ROS sino también de reforzar las defensas naturales de la piel y evitar la inflamación y resequedad de la misma.

La mayoría de los niños permanecen alrededor de un $80 \%$ de su vida bajo el sol antes de los 18 años de edad, y por esto se confirma que más de la mitad de la exposición solar ocurre dentro de los primeros veinte años de vida, de allí que sea importante que los padres enseñen a sus hijos a disfrutar del sol en forma segura.

\section{OBJETIVOS}

En conocimiento de que la resequedad de la piel es una de las sensaciones mas tratadas por Dermatólogos, esteticistas y consumidores individualmente, nọs propusimos comparar el efecto hidratante de una solución acuosa de Proteína de soja (Glycine hispida), con una sustancia de origen vegetal como el Aloe o
Sábila (Aloe barbadenses), de cuyas hojas se extrae una sustancia resinosa llamada acíbar, que corresponde a un glucósido de color amarillo verdoso tornándose más oscuro al contacto con el aire de propiedades hidratantes ampliamente conocidas.

Asimismo, se comprobaron el efecto antiprurriginoso de la fracción lipídica de la cebada, obtenida en el proceso de elaboración de la cerveza, con un placebo que contenía áloe vera y extracto de manzanilla.

Y finalmente, diseñar la formulación de un bloqueador solar con filtros físicos, con un complejo de proteínas de soja como hidratante, antiirritante y con actividad antiozono, y el residuo lipídico de la cebada como antiinflamatorio y antipruriginoso, determinando su efectividad mediante un estudio clínico.

\section{MATERIALES Y MÉTODOS}

\section{Efecto hidratante de la proteína de Soja}

La medida de la hidratación fue realizada mediante un Corneómero CM 825. Esta medida se basa en la capacidad de la epidermis de indicar el contenido de agua de la capa cómea. El valor bajo corresponde a la piel seca y los valores altos a una piel muy hidratada. La prueba registra la capacidad de cambios causados por diferentes contenidos de humedad de la piel. Se realizan 4 mediciones continuas en varias áreas de la piel. El promedio resultante se observa en la pantalla del aparato. Puede mostrarse la cinética de la humedad de varios productos durante un período de 2 horas contra el aumento de humedad en términos de porcentaje. El valor de la hidratación depende de la condición ambiental del cuarto donde se realice el estudio clínico. Básicamente debe realizarse en condiciones de temperaturas entre $24-26^{\circ} \mathrm{C}$ y $48-52 \%$ de humedad relativa $K$. (19) (20). 
En este sentido, siguiendo las normas de formulación de Productos Cosméticos (3), se diseñaron varias formulaciones, seleccionando una base gelificada para incorporar a la solución acuosa de la proteína de soja y el extracto de Aloe vera, la cual se sometió a pruebas de estabilidad física a diferentes temperaturas y se comprobó su inocuidad microbiológica. Se incorporó el extracto acuoso de proteína de soja al 5\% a la base hipoalergénica (sin fragancia ni colorantes). Con el áloe vera se procedió de igual forma, es decir al $5 \%$ y se rotularon como:

Hidratante "A", con $5 \%$ de extracto de áloe vera.

Hidratante "B", con 5\% de sol acuosa de proteína de soja. (cuadro 1)

El estudio se realizó con 20 pacientes del sexo femenino, con edades comprendidas entre los 35 y 60 años, que presentaban piel normal y reseca.

Se les indico no utilizar ningún tipo de cosméticos durante las 24 horas previas al estudio, y lavarse el antebrazo con solución acuosa al $10 \%$ de lauril sulfato de sodio y posterior aplicación de etanol. Se dejaron aclimatar por 30 minutos en el cuarto donde se realizó el estudio. Posteriormente, se les indicó aplicarse en dos zonas del antebrazo en un espacio de 4 x $5 \mathrm{~cm}$, cada una de las dos preparaciones. Se dejaron secar y se realizaron las medidas en el Corneómetro a los $0,30,60$, 90,120 y 180 minutos.

En el otro antebrazo se procedió de la misma forma, pero con un placebo.

La base hipoalergénica en gel con el extracto vegetal estuvo formado por: agua $93,8 \%$, extracto vegetal (Sol. acuosa de proteína de soja o Ext. de áloe vera) 5\%, polímeros del ácido acrílico $0,7 \%$, trietanolamina $0,3 \%$,parabenos-imidazolidinil urea $0,2 \%$.

En el Placebo, él \% de extracto vegetal, se reemplazó por agua.

Cuadro 1. Composición del hidratante A y B

\begin{tabular}{|l|c|c|}
\cline { 2 - 3 } \multicolumn{1}{c|}{} & $\%$ & $\%$ \\
\cline { 2 - 3 } \multicolumn{1}{c|}{} & $\begin{array}{c}\text { Hidratante } \\
\mathrm{A}\end{array}$ & $\begin{array}{c}\text { Hidratante } \\
\mathrm{B}\end{array}$ \\
\hline Pólimeros del ácido acrílico & 0.7 & 0.7 \\
\hline Trietanolamina & 0.3 & 0.3 \\
\hline Ext. de aloe vera & 5 & -- \\
\hline Sol. acuosa proteína soja & -- & 5 \\
\hline Agua c.s.p & 100 & 100 \\
\hline
\end{tabular}

Actividad antipruriginosa del residuo lipídico de la cebada

De las diferentes formulaciones se seleccionó la más adecuada para la realización del estudio clínico en pacientes con prurito por el uso de productos cosméticos como antitranspirantes, despigmentantes, depilatorios, jabones, después de una exposición solar exagerada y después del rasurado. Para ello se utilizó además de la observación clínica para evaluar la mejoría (individualmente variable), un Mexameter MX 16 que midió los siguientes parámetros: disminución de la intensidad eritemal (consecuencia del prurito) y la recuperación de las alteraciones dermatíticas existentes.

El Mexameter MX 16 es un equipo que suministra una determinación exacta, rápida y reproducible del Indice del Eritema, mediante la medida de la hemoglobina. La medida se basa en el principio de la absorción. El electrodo emite una luz de 3 longitudes de onda conocidas. Un receptor mide la luz reflejada en la piel. Las posiciones tanto del emisor como del receptor garantizan que solamente se midan la luz difusa y dispersa. 
Para la evaluación del eritema se usan dos longitudes de onda para medir la capacidad de absorción de la piel. Una de estas longitudes de onda corresponde al pico de absorción espectral correspondiente a la hemoglobina. La otra longitud de onda, ha sido escogida para evitar influencias de color (bilirrubina). El proceso de medición comienza automáticamente, colocando el electrodo y presionándolo suavemente en posición recta sobre la superficie de la piel (22).

La fracción lipídica de la cebada es obtenida mediante filtración, durante el proceso de fermentación para la fabricación de la cerveza. El proceso de extracción mediante $\mathrm{CO} 2$ corresponde a un proceso suave $\mathrm{y}$ ecológico, utilizando hoy en día en numerosas investigaciones. Los granos del residuo son extraídos a una temperatura de $60^{\circ} \mathrm{C}$ bajo alta presión y con la exclusión de oxígeno. Al retornar a la presión atmosférica se obtiene un producto totalmente libre de solventes. Esta sustancia corresponde a una cera amarilla dorada, con el agradable olor típico de los cereales y que corresponde al ingrediente activo de esta investigación (13).

De la fracción lipídica de la cebada, se utilizó a la concentración del $5 \%$, de acuerdo a la literatura científica (13).

Siguiendo los principios básicos generales sobre la formulación de Productos Cosméticos (3), se diseñó una formulación de acuerdo a nuestros objetivos, fundamentados en la acción del agente activo antirpruriginoso, obtenido de la fracción lipídica de la cebada y la selección de la base cosmética adecuada.
Se formularon varias emulsiones: o/w y w/o, de diferentes viscosidades, observando la facilidad de incorporación del ingrediente activo, la facilidad de aplicación, y la factibilidad de adquisición en Venezuela y por supuesto, el costo del producto final.

Se formularon 2 productos en crema que se denominaron "EMULSION A" $\mathrm{y}$ "EMULSION B" (cuadro 2).

Ambas cremas tenían como base emulsión no iónica con agente de cuerpo, emulsificantes y preservativos.

50 de ellas se rotularon como "EMULSION B" con $2,5 \%$ de Ext. de áloe vera y $2,5 \%$ de Ext. de manzanilla.

50 de ellas se rotularon como "EMULSION A" con el agregado de $5 \%$ de la fracción lipídica de la cebada.

Cuadro 2. Formulación de dos productos en crema

\begin{tabular}{|l|c|c|}
\hline & $\begin{array}{c}\% \\
\text { Emulsión } \\
\mathrm{A}\end{array}$ & $\begin{array}{c}\% \\
\text { Emulsión } \\
\mathrm{B}\end{array}$ \\
\hline Fracción lipídica cebada & 5 & -- \\
Ext. áloe vera & -- & 2,5 \\
Ext. manzanilla & -- & 2,5 \\
Gliceril estearato & 10 & 10 \\
Alcohol cetílico & 5 & 5 \\
Ceteareth-12 & 1,5 & 1,5 \\
Ceteareth-20 & 1,5 & 1,5 \\
Propilenglicol & 5 & 5 \\
Preservativos & $\mathrm{c.s}$ & $\mathrm{c.s}$ \\
Agua C.S.P & 100 & 100 \\
\hline
\end{tabular}

Posteriormente, se sometió el producto final a pruebas de estabilidad física a diferentes temperaturas $\left(10-15^{\circ} \mathrm{C}\right.$ en la nevera, $27-30^{\circ} \mathrm{C}$ temperatura ambiente y $35-40^{\circ} \mathrm{C}$ en la estufa), 
y también se comprobó la inocuidad microbiológica, siguiendo la metodología del Microbiological Methods for Cosmetics (FDA) (21).

Los estudios clínicos fueron doble ciego, en 30 pacientes que presentaban prurito, con edades comprendidas entre 10 y 45 años. Se evaluó la Ohistoria clínica del paciente y su tratamiento. Se les indicó la aplicación de dos veces diarias con emulsiones cremosas que se denominaron EMULSION "A" para un lado del cuerpo (con fracción lipídica de la cebada al 5\%) y EMULSION "B" (con ext. de Aloe vera y Manzanilla), para otro lado.

Se estudiaron los siguientes parámetros:

- Disminución de la intensidad eritemal, consecuencia del prurito, mediante un aparato Mexamenter MX 16 y

- La recuperación de las alteraciones dermatíticas existentes, y

- La mejoría del prurito por observación clínica del médico y opinión del propio paciente.

- Estas mediciones se realizaron durante un periodo de 3 semanas.

\section{Medida de la efectividad del protector solar}

Siguiendo los principios básicos de formulación de Productos Cosméticos 83), se diseñó una formulación que contenía como ingredientes activos: Oxido de zinc y dióxido de titanio (filtros físicos), un complejo de proteína de soja (hidratante, antiirritante y con actividad antiozono) y residuo lipídico de cebada (antipruriginoso y antiinflamatorio) (cuadro 3).
Cuadro 3. Formulación del protector solar

\begin{tabular}{|l|c|}
\hline \multicolumn{1}{|c|}{ EMULSION } & $\%$ \\
\hline Oxido de zinc & 5 \\
Dióxido de titanio & 7 \\
Complejo de proteína de soja & 2 \\
Extracto de cebada & 3 \\
Gliceril estearato & 10 \\
Alcohol cetílico & 5 \\
Ceteareth-12 & 1,5 \\
Ceteareth-20 & 1,5 \\
Propilenglicol & 5 \\
Preservativos & c.s \\
Agua C.S.P & 100 \\
\hline
\end{tabular}

Se realizó un estudio clínico con 25 pacientes de edades comprendidas, entre los 16 y 35 años, de piel tipo I y II, a los cuales se les determinó la intensidad eritemal (mẹediante un Mexameter MX 16 (19), la dosis mínima eritemal (mediante el Sun Protection Diagnostic SP37 (19) y el estado de hidratación en el área de la cara (mediante un Corneómetro CM825 (19). Ya se ha mencionado que el Mexameter MX 16 indica determinaciones exactas, rápidas y reproducibles del color de la piel y de su condición.

El Sun Protection Diagnostic SP37 nos suministró el valor de la dosis mínima eritemal, mediante la medida del contenido de melanina de la piel. A su vez es capaz de indicar cual es el factor de protección solar adecuado para la persona y el tiempo máximo de exposición solar de acuerdo a la ubicación geográfica y al tipo de piel (19).

Posteriormente, se les aplicó la formulación objeto de este estudio y se les indicó exponerse a la radiación solar en horas del medio día en un playa de Caracas, por un tiempo superior a 15 veces su DME, reaplicando el producto en caso de sudoración. Al cabo de este tiempo se midió de nuevo la intensidad eritemal, obteniendo los siguientes 
resultados: el $98 \%$ de los pacientes no presentaban aumento de la intensidad eritemal, ni manifestaron tener prurito.

En cuanto a la hidratación, se encontró que el 96\% de los sujetos mantenían su piel bien hidratada, mientras que el $4 \%$ restante presentó una ligera disminución en las lecturas del Corneómetro.

\section{RESULTADOS}

1. En relación al efecto hidratante de la proteina de soja se encontraron los siguientes resultados. (cuadro 4)

Cuadro 4. Medida de Hidratación en el corneómetro

\begin{tabular}{|c|c|c|c|}
\hline $\begin{array}{c}\text { Tiempo en } \\
\text { minutos }\end{array}$ & $\begin{array}{c}\text { Valor } \\
\text { placebo }\end{array}$ & $\begin{array}{c}\text { Valor áloe } \\
\text { vera }\end{array}$ & $\begin{array}{c}\text { Valor } \\
\text { soya }\end{array}$ \\
\hline 0 & 35 & 35 & 40 \\
30 & 58 & 63 & 89 \\
60 & 51 & 62 & 79 \\
90 & 43 & 52 & 77 \\
120 & 43 & 50 & 72 \\
180 & 33 & 47 & 72 \\
\hline
\end{tabular}

\section{En relación a la actividad antipruriginosa}

del residuo lipídico de la cebada, tenemos que de acuerdo al seguimiento observado durante tres meses para las pruebas de estabilidad física, a diferentes temperaturas que variaban entre $10-40^{\circ} \mathrm{C}$, se encuentra que ambas emulsiones permanecieron estables.

En cuanto al estudio de inocuidad microbiológica se reporta que las muestras objetivos del estudio fueron sometidas a estudio microbiológico de acuerdo al Microbiological Methods for Cosmetics (FDA), obteniéndose los resultados mostrados en el cuadro 5.
Cuadro 5. Actividad antipruriginosa del residuo lipídico de la cebada

\begin{tabular}{|l|c|}
\hline Bacterias aerobias mesófilas & $<$ de 10 \\
\hline Mohos (ufc/g) & $<$ de 10 \\
\hline Levaduras (ufc/g) & $<$ de 10 \\
\hline Staphylococcus aureus & Ausente \\
\hline Enterobacterias & Ausente \\
\hline Pseudomonas & Ausente \\
\hline
\end{tabular}

En el estudio clínico de los 30 pacientes que recibieron el tratamiento indicado mediante la aplicación dos veces diarias de emulsiones cremosas denominadas EMULSION A (con fracción lipídica de la cebada al 5\%) para un lado del cuerpo y EMULSION B (con ext. de áloe vera y manzanilla), para el otro lado, se obtuvieron los resultados que figuran en los cuadros 6 y 7 .

Cuadro 6. Con la Emulsión "A"

\begin{tabular}{|l|c|}
\hline \multicolumn{1}{|c|}{ Evaluación } & $\mathrm{N}^{\circ}$ pacientes \% \\
\hline $\begin{array}{l}\text { Disminución de la } \\
\text { Intensidad eritemal }\end{array}$ & 77 \\
\hline Reducción del Prurito & 80 \\
\hline $\begin{array}{l}\text { Recuperación de } \\
\text { Alteraciones } \\
\text { Dermatíticas }\end{array}$ & 75 \\
\hline Preferencia producto & 100 \\
\hline
\end{tabular}

Cuadro 7. Con la Emulsión "B"

\begin{tabular}{|l|c|}
\hline \multicolumn{1}{|c|}{ Evaluación } & $\mathrm{N}^{\circ}$ pacientes \% \\
\hline $\begin{array}{l}\text { Disminución de la } \\
\text { Intensidad eritemal }\end{array}$ & 40 \\
\hline Reducción del Prurito & 50 \\
\hline $\begin{array}{l}\text { Recuperación de } \\
\text { Alteraciones } \\
\text { Dermatíticas }\end{array}$ & 27 \\
\hline Preferencia producto & 0 \\
\hline
\end{tabular}


3. En relación a los resultados obtenidos en la determinación de la efectividad del protector solar se muestran en el cuadro 8.

Cuadro 8. Determinación de la efectividad del protector solar.

\begin{tabular}{|l|c|}
\hline \multicolumn{1}{|c|}{ Emulsión } & $\%$ \\
\hline $\begin{array}{l}\text { Sin aumento de la } \\
\text { intensidad eritemal }\end{array}$ & 98 \\
\hline Sin prurito & 98 \\
\hline Estado de hidratación & 96 \\
\hline $\begin{array}{l}\text { Baja lectura en } \\
\text { Corneómetro }\end{array}$ & 4 \\
\hline
\end{tabular}

\section{CONCLUSIONES}

Sobre la base de los resultados obtenidos podemos concluir en que:

1. La Proteína de Soja mantiene el efecto hidratante más tiempo que el Aloe vera (inclusive por más de 3 horas), por lo tanto puede recomendarse su incorporación en productos cosméticos como sustancia hidratante de origen natural, segura y efectiva.

2. El residuo lipídico de la Cebada, presenta actividad antipnuriginosa significativa; observándose disminución de la intensidad eritemal y recuperación de las alteraciones dermatiticas. Por lo tanto se recomienda incorporar en productos para y después de la exposición solar y del afeitado, depilatorios, despigmentantes, en jabones, etc. ya que constituye una alternativa válida y segura para la prevención del prurito.
3. La formulación del protector solar con la combinación de filtros físicos $(\mathrm{OZn} y$ TiO2) y extractos vegetales como el Complejo de Proteína de Soja y el Residuo Lipídico de la Cebada (de acción antiinflamatoria-antipruriginosa) pueden prevenir las mutaciones genéticas que supuestamente originan el cáncer de piel tipo no-melanoma y el fotoenvejecimiento, $y$ ofrecen un camino seguro y efectivo para el tratamiento de la piel dañada.

\section{REFERENCIAS BIBLIOGRÁFICAS}

1. Vargas, A., Castro, A. 1999 Proteína de soja: Evaluación de su efecto hidratante, Libro de Trabajos científicos y Posters del XIV Congreso Latinoamericano e Ibérico de Químicos Cosméticos \& I.F.S.CC International Conference, págs. 247-252.

2. Rojas, C., Castro, A., Castro, L., Brito, R. 1999 Utilización del residuo lipídico de la cebada en el tratamiento del prurito, Libro de Trabajos científicos y Posters del XIV Congreso Latinoamericano e Ibérico de Químicos Cosméticos \& I.F.S.CC International Conference, págs. 127-134.

3. Castro A. de 1987 Principios Básicos de Formulaciones Cosméticas. Ed. A. de Castro, Caracas.

4. Wachter, R., Salka, B., y Magnet, A. 1997 Fitosteroles: Presencia de sustancias activas de origen vegetal en cosméticos, Cosméticos Nuevos, 1, págs. 38-42.

5. Bhaattacharyya, 1983 WE Connor and DS.Lin, J. Invest. Dermatol., 80: págs. 294-296.

6. Hager. 1950 Tratado de Farmacia Práctica, Editorial Labors, Tomo I, 9.1.115. 
7. Pentapharm Ltd.1998 Literatura científica sobre el Elhibin.

8. Soucre, L. 1996 Prurito, en Dermatología Rondón Lugo, Tomo 1, Cap.40, Ed. Reinaldo Godoy, Caracas p. 431.

9. Piquero, J.; Castro, A. 1996 Erupciones inducidas por cosméticos en Guía Dermocosmética de Venezuela, Cap. 23, Ed. Grupo P1CAS, Caracas, págs. 90-91.

10. Da Fonseca, A.; Nogueira, P., L. 1993 Fisioterapia Dematológica: banhos e balneoterapia, en Manual de Terapéutica Dermatológica en Dermatología, Ed. Livraria Roca LTDA, Sao Paulo, págs. 251-259.

11. Grijalbo 1986 Enciclopedia Grijalbo, Gamilia Gramineas, Ediciones Grijalbo S.A. Tomo 1, p. 403.

12. Schnee L. 1984 Plantas comunes en Venezuela. Tercera edición. Universidad Central de Venezuela; Caracas p. 806.

13. Perntapharm 1996 LTD, Literatura científica sobre el Stimutex.

14. Cáceres, A. 1996 Plantas de uso medicinal en Guatemala. Editorial Universitaria; Ciudad de Guatemal p. 402.

15. Duke, J.A. y Atchley, A.A. 1986 CRC handbook of proximate analysis tables of higher plants. CRC Press. Inc. Baton Rouge (7), p. 389.
16. McPherson H.C. 1998 Health New. Sunscreen \& Cancer New England Journal of Medicine. 1998. by ¿Does Sunscreen Really Protect Against Skin Cancer? 1998. Roach M., L. Getting serious about skin cancer.

17. Lasch J, Schoenfelder U, Walke M, $\begin{array}{llll}\text { Zellmer S, } & \text { Becker } & \text { D. } & 1998\end{array}$ Photooxydative damage pf skin lipids in liposomes (hSCLLs): interference of sterols with the lipids peroxidation chain International Journal Pharmacy, 162: págs. 126-136.

18. Scharffetter-kochanek-Kochanek K, Wlaschek M, Breiinneisen P, Schaun $M$, Blaudschum R, Wenk J. 1997 UVinduced reactive oxygen speciesin photocarcinogenesis and photoaging Biological Chemistry, 378: págs. 12471257.

19. Schrader K. 1997 ¿Podemos medir la efectividad de los cosméticos? Ciencia y Cosmética, 114, 21-25.

20. Muller, P. 1998 The Skin: subject and object of sensory perception, 1, págs. 39-47.

21. F.D.A. 1992 Microbiological Methods for Cosmetics, Bacteriological Analitical Manual.

22. Corugage Khazaca 1997 Electronic $\mathrm{GmbH}$, Información Técnica. 